Check for updates

Cite this: Nanoscale Adv., 2019, 1, 3279

\title{
Infrared tunable, two colour-band photodetectors on flexible platforms using 0D/2D PbS-MoS 2 hybrids $\uparrow$
}

\author{
S. Mukherjee, (D) ${ }^{a}$ S. Jana, ${ }^{a}$ T. K. Sinha, ${ }^{a}$ S. Das ${ }^{b}$ and S. K. Ray (D) *ac
}

Two-dimensional (2D) $\mathrm{MoS}_{2}$ nanosheets have been integrated with zero-dimensional (OD) PbS quantum dots to achieve a superior optical response extending to the short-wavelength infrared region along with a broadband visible response for multispectral photodetection. The 0D/2D hybrid nanostructures have been synthesized by a one pot, stabilizer-free solvothermal growth process. Microscopic and spectroscopic studies confirmed the formation of PbS QD decorated semiconducting $2 \mathrm{H}-\mathrm{MoS}_{2}$ layers. The size tunable absorption features with longer photo-generated carrier lifetime of synthesized hybrid nanostructures indicate that the integration of $\mathrm{PbS} Q \mathrm{QDs}$ in $\mathrm{MoS}_{2}$ could be a viable approach for fabricating two-colour band photodetectors, viz. visible broadband and wavelength selective short-wave IR photodetectors. Devices have also been demonstrated on polyethylene terephthalate substrates using a solution-based synthesis technique for flexible and ultrathin optoelectronic device applications. The photodetection performance of fabricated devices suggests that the synergic 0D/2D hybrid nanostructures are significantly superior to solution processed hybrid devices operating in the infrared region. The successful integration of $O D$ QDs in 2D materials may pave the way for novel, high performance, next-generation CMOS compatible flexible photonic devices.

Received 15th May 2019

Accepted 10th July 2019

DOI: 10.1039/c9na00302a

rsc.li/nanoscale-advances
On the other hand, several intriguing properties of leadchalcogenide $(\mathrm{PbX})$ nanocrystals (NCs), such as tunable infrared (IR) absorption owing to the quantum confinement and relatively larger excitonic Bohr radius, have inspired researchers to incorporate them into $2 \mathrm{D}$ materials to achieve higher efficiency with an extended absorption edge in the solar spectrum. Recently, hybrid graphene/PbS quantum dot (QD) phototransistors, ${ }^{15,16} \mathrm{PbS} / \mathrm{CNT}$ nanohybrids ${ }^{17}$ and $\mathrm{PbS} /$ $\mathrm{MoX}_{2}{ }^{18-20}$ phototransistors have been demonstrated as promising infrared-photovoltaic devices. However, the synthesis of $\mathrm{PbS}$ QDs for device fabrication involved complicated and hazardous chemical pathways using different reducing agents, stabilizing agents, eco-hazard sulfur sources and a complex lithography process, limiting their widespread applications. ${ }^{19-21}$ On the other hand, an in situ synthesis approach for the $\mathrm{MoS}_{2}-$ $\mathrm{PbS}$ hybrids appears very attractive, since the large surface area of $2 \mathrm{D}$ materials not only acts as an ideal template for the nucleation and growth of QDs but also offers them enhanced stability due to metal-sulfur interactions in the case of $\mathrm{MoS}_{2}$ (e.g., $\mathrm{M}^{n+}$ with $\mathrm{S}^{-}$or vacant $\mathrm{d} / \mathrm{f}$-orbitals of metal with the available lone pair of electrons of sulfur), etc. This provides a synergistic effect in QD decorated 2D materials, ${ }^{22,23}$ leading to efficient photo-induced charge carrier separation in QDs and concomitant carrier transport in 2D nanosheets, making the hybrid $0 \mathrm{D} / 2 \mathrm{D}$ heterostructure a potential candidate for developing next-generation high-performance optoelectronic devices. ${ }^{18-21,24}$ Apart from the facile processing approach and 
improved properties, the compatibility of the fabrication process with standard CMOS technology is a crucial need for practical applications.

Here we demonstrate the realization of superior performance of two colour-band (visible broadband and tunable short-wave infrared) photodetectors in a single device, by integrating PbS QDs with $\mathrm{MoS}_{2}$ nanosheets using a facile and in situ synthesis approach. The size dependent optical absorption of $0 \mathrm{D} / 2 \mathrm{D} \quad \mathrm{PbS} / \mathrm{MoS}_{2}$ hybrid heterostructures and their longer photo-carrier lifetime are attractive for improved broadband and wavelength tunable IR photodetection. Further, the one pot chemical technique allows the synthesis of $\mathrm{PbS}$ QD decorated few-layer $2 \mathrm{D} \mathrm{MoS}$ nanosheets integrated on polyethylene terephthalate (PET) substrates paving the way for the realization of photodetectors for next-generation flexible and ultrathin photonic devices using $0 \mathrm{D} / 2 \mathrm{D}$ hybrid heterostructures.

\section{Experimental}

\subsection{Synthesis of hybrid nanostructures}

Bulk molybdenum disulfide $\left(\mathrm{MoS}_{2}\right)$ powder and lead acetate $\left(\mathrm{Pb}(\mathrm{Ac})_{2}\right)$ (Sigma-Aldrich) were used without further purification. One-pot synthesis of PbS QD incorporated few-layers $\mathrm{MoS}_{2}$ (FLM), without the use of any external reducing agents or stabilizers, was performed by dispersing $100 \mathrm{mg}$ of $\mathrm{MoS}_{2}$ powder in $50 \mathrm{~mL}$ aqueous $N, N$-dimethylformamide (DMF) (45 mL DMF and $5 \mathrm{~mL} \mathrm{H}_{2} \mathrm{O}$ ) under mild stirring for $30 \mathrm{~min}$. This was followed by the addition of catalytic $\mathrm{Pb}(\mathrm{Ac})_{2}$ to the $\mathrm{MoS}_{2}$ dispersion and stirring for $30 \mathrm{~min}$ to get a homogeneous mixture. Thereafter, the mixture was transferred into an $80 \mathrm{~mL}$ Teflon-lined stainless steel autoclave chamber and the autoclave was placed in a pre-heated furnace to accomplish the solvothermal reaction at $150{ }^{\circ} \mathrm{C}$ for $2 \mathrm{~h}$ to obtain in situ grown $\mathrm{PbS}$ decorated $\mathrm{MoS}_{2}$ nanosheets. Step-by-step schematic illustration of the synthesis of $\mathrm{PbS}$ QDs/ $\mathrm{MoS}_{2}$ hybrid nanostructures and their synthesis mechanism are presented in Fig. S1 (see ESI $\dagger$ ). DMF played a dual role working as an exfoliating solvent for bulk $\mathrm{MoS}_{2}$, as well as the reduction agent for $\mathrm{Pb}$ ions. On the other hand, the metal salt (i.e., Pb-acetate) acted as an intercalating agent (Pb-ion) which facilitated the successful exfoliation of $\mathrm{MoS}_{2}$ using $\mathrm{Pb}$-ions, instead of alkali-metal ions for the first time. The donor-acceptor interactions among the S-atoms of $\mathrm{MoS}_{2}$ and metal-ions of $\mathrm{Pb}(\mathrm{Ac})_{2}$ favored the intercalation during sonication. At the time of solvothermal reaction, DMF was instrumental in reducing $\mathrm{Pb}^{2+}$ to a more reactive $\mathrm{Pb}^{0}$, which in turn reacted with the interfacial sulfur of the $\mathrm{MoS}_{2}$ layer, resulting in sulfidation of $\mathrm{Pb}^{0}$ to $\mathrm{PbS}$.

In our novel synthesis process, the $\mathrm{PbS}$ quantum dots need not be passivized using any chemically hazardous stabilizers or capping agents, such as ETD, oleylamine, oleic acid, etc., as reported by others. In the proposed synthesis technique the $\mathrm{PbS} / \mathrm{MoS}_{2}$ hybrid heterojunction was formed with an increased interfacial area, where $\mathrm{MoS}_{2}$ itself served as a capping layer of $\mathrm{PbS}$, leading to the formation of clean and defect-free $\mathrm{PbS} / \mathrm{MoS}_{2}$ interfaces required for devices with high external quantum efficiency. The solution processable hybrid material would facilitate the integration on an almost limitless variety of substrates readily by spin-coating, spray-casting, or inkjetprinting leading to its applications in large area, low cost, flexible optoelectronic devices. After removing the reaction chamber from the furnace, the mixture was cooled to roomtemperature followed by ultrasonication for $15 \mathrm{~min}$, which provided sufficient mechanical energy to overcome van der Waals forces of $\mathrm{MoS}_{2}$ leading to randomly distributed PbS QDS on exfoliated $\mathrm{MoS}_{2}$ nanosheets to form hybrid nanostructures. The resultant dispersion was centrifuged first for $15 \mathrm{~min}$ at $1000 \mathrm{rpm}$ to remove the unreacted inorganic materials. The precipitate was collected, re-dispersed in DMF and sonicated for $10 \mathrm{~min}$. Then the dispersion was centrifuged at $5000 \mathrm{rpm}$ for $15 \mathrm{~min}$ to separate the intercalated lighter $\mathrm{PbS} / \mathrm{MoS}_{2}$ hybrid as the supernatant. After diluting the remnant part with DMF, the dispersion underwent centrifugation for another 3 times under similar conditions. Collected supernatants were mixed under mild sonication for $10 \mathrm{~min}$. The final yellowish solution thus obtained was used for the measurements and device fabrication. Hybrid heterostructures with various lead acetate concentrations (namely $1 \mathrm{mg}, 5 \mathrm{mg}, 10 \mathrm{mg}$ and $25 \mathrm{mg}$ ) were synthesized using the same procedure keeping the amount of $\mathrm{MoS}_{2}$ fixed $(100 \mathrm{mg})$ and resultant samples are hereafter referred to as S1, S2, S3 and S4, respectively. Pristine $\mathrm{MoS}_{2}$ layers without $\mathrm{PbS}$ quantum dots were also synthesized as control samples using the standard ion-intercalation method reported previously. ${ }^{25}$

\subsection{Device fabrication \& measurement set-up}

Batch processing of large area metal-semiconductor-metal (MSM) devices was carried out using a standard photolithography process on flexible and transparent PET substrates, followed by subsequent metal (Au) electrode deposition. PET substrates were coated with a photoresist (AZ 5214E) and exposed for 7 seconds under monochromatic UV light (wavelength: $355 \mathrm{~nm}$ ) to transfer the mask pattern, followed by dipping in a developer for $25 \mathrm{~s}$ and thoroughly washed in deionized (DI) water. Subsequent metal $(\mathrm{Au} \sim 80 \mathrm{~nm}$ ) electrode deposition was done by thermal evaporation. Finally, warm acetone was utilized for the lift-off of the photoresist and drying under nitrogen purging. The fabrication of photodetectors was completed by drop casting of as-synthesized PbS-QDs/ $\mathrm{MoS}_{2}$ hybrid nanostructures on the channel sandwiched between the Au electrodes on PET substrates followed by drying at normal ambient temperature. The current-voltage $(I-V)$ characteristics were recorded using a Keithley 2400 source meter. The photoresponse of devices was measured under visible to infrared irradiation by combining a broadband source and a calibrated monochromator. The light excitation was modulated using a mechanical chopper at regular time intervals to study the time-resolved photoresponse of the device as an optical switch.

\section{Results and discussion}

\subsection{Properties of $\mathbf{P b S}-\mathrm{MoS}_{2}$ hybrids}

The typical high-resolution FESEM image of $\mathrm{PbS} / \mathrm{MoS}_{2}$ hybrid nanostructures with a precursor wt ratio of $1: 100$ of $\mathrm{PbS}$ to 
$\mathrm{MoS}_{2}$ (sample S1) is shown in Fig. 1(a). On the other hand, the TEM micrograph of the as-prepared $\mathrm{PbS} / \mathrm{MoS}_{2}$ hybrid nanostructure (sample S1) is depicted in Fig. 1(b). The inset of Fig. 1(b) represents a typical TEM micrograph of few layer bare $\mathrm{MoS}_{2}$ nanosheets (control sample) with an average lateral size of $\sim 300 \mathrm{~nm}$. Both the micrographs reveal the presence of nearly spherical shape mono-dispersed PbS QDs uniformly distributed over a large area on the few-layers $\mathrm{MoS}_{2}$ surface. The sizedistribution of $\mathrm{PbS}$ QDs indicates the formation of variable size QDs ranging from 5-10 $\mathrm{nm}$ with an average size of $\sim 8 \mathrm{~nm}$. The size is well below the Bohr-excitonic radius of $\mathrm{PbS}(\sim 20$ $\mathrm{nm}) .{ }^{26}$ The size of PbS QDs is found to increase with increasing molar concentration of $\mathrm{Pb}(\mathrm{Ac})_{2}$ with an average size of $\sim 12 \mathrm{~nm}$, $\sim 18 \mathrm{~nm}$ and $\sim 25 \mathrm{~nm}$ for samples S2, S3 and S4, respectively. All $\mathrm{PbS}$ QDs are found to be attached to $\mathrm{MoS}_{2}$ nanosheets due to the presence of a large number of unsaturated dangling bonds and active sites (sulfur vacancies) at the $\mathrm{MoS}_{2}$ surface, resulting in nucleation and subsequent growth. Moreover, an enormous surface area of $\mathrm{MoS}_{2}$ nanosheets plays the role of a stabilizer that hinders the agglomeration of PbS QDs. The typical AFM micrograph of the as-synthesized bare $\mathrm{MoS}_{2}$ nanosheets is exhibited in Fig. S2 (please see ESI $\dagger$ ). The average thickness of the $\mathrm{MoS}_{2}$ nanosheets is found to be $\sim 8 \mathrm{~nm}$, with average lateral dimensions between 200 and $300 \mathrm{~nm}$, confirming the formation of two-dimensional (2D) few-layer $\mathrm{MoS}_{2}$.

The elemental composition and the nature of chemical bonding of $\mathrm{PbS} / \mathrm{MoS}_{2}$ hybrid nanostructures were investigated by XPS and AES spectroscopy. Fig. 2(a) presents a selected area FESEM micrograph of sample S1, where the AES mapping has been acquired. Fig. 2(b), (c) and (d) show the individual elemental mapping images of $\mathrm{Mo}, \mathrm{S}$ and $\mathrm{Pb}$ atoms, respectively, indicating the formation of a $\mathrm{PbS} / \mathrm{MoS}_{2}$ hybrid. The combined AES mapping for all three elements (Mo, $\mathrm{Pb}$ and $\mathrm{S}$ ), presented in Fig. 2(e), reveals their homogeneous distribution in the hybrid material. High-resolution AES spectra are presented in Fig. 2(f), showing the presence of Mo KLL, S KLL and Pb KLL transition lines in the sample. The atomic ratio (\%) of the individual elements present in the hybrid is shown in the inset of Fig. 2(f). A slight Pb-richness in $\mathrm{PbS} / \mathrm{MoS}_{2}$ hybrids is attributed to the presence of $\mathrm{Pb}$ on the surface due to its low surface diffusivity and the existence of $S$ vacancies. The chemical bonding configuration of synthesized $\mathrm{PbS} / \mathrm{MoS}_{2}$ hybrids has been

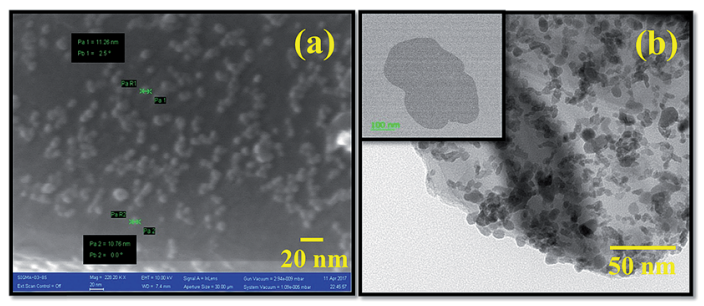

Fig. 1 (a) High-magnification FESEM image of the as-synthesized PbS/ $\mathrm{MOS}_{2}$ hybrid nanostructures for sample-S1. (b) Typical TEM micrographs of the as-synthesized $\mathrm{PbS}_{\mathrm{MoS}}$ hybrid samples (sample-S1), showing a large number of well dispersed QDs attached on the $\mathrm{MoS}_{2}$ surface. Inset of (b) exhibits bare $\mathrm{MoS}_{2}$ nanosheets synthesized by the ion-intercalation method. studied by XPS spectroscopy using Al-K $\alpha$ radiation of energy $1.4866 \mathrm{keV}$. High resolution XPS spectra of Mo, S and Pb corelevel electrons are depicted in Fig. S3(a), (b) and (c), respectively (please see ESI $\dagger$ ). The well-resolved characteristic doublets for Mo $3 \mathrm{~d}\left(3 \mathrm{~d}_{5 / 2}\right.$ and $\left.3 \mathrm{~d}_{3 / 2}\right), \operatorname{Pb} 4 \mathrm{f}\left(4 \mathrm{f}_{7 / 2}\right.$ and $\left.4 \mathrm{f}_{5 / 2}\right)$, and $\mathrm{S} 2 \mathrm{p}\left(2 \mathrm{p}_{3 / 2}\right.$ and $S 2 p_{1 / 2}$ ) are in excellent agreement with their reported values, confirming the formation of $\mathrm{Mo}^{4+}, \mathrm{Pb}^{2+}$ and $\mathrm{S}^{2-}$ states, respectively. ${ }^{27-29}$ The shift of binding energy peaks of $\mathrm{S} 2 \mathrm{p}(\sim 2.4$ $\mathrm{eV})$, Mo 3d $(\sim 1 \mathrm{eV})$ and $\mathrm{Pb} 4 \mathrm{f}(\sim 1 \mathrm{eV})$ electrons with respect to their elemental values reveals the formation of $\mathrm{Mo}-\mathrm{S}$ and $\mathrm{Pb}-\mathrm{S}$ chemical bonds, supporting the formation of $\mathrm{PbS}$ decorated $\mathrm{MoS}_{2}$ nanosheets.

XRD patterns of synthesized samples with different precursor ratios (S1-S4) are presented in Fig. 3(a). Duly indexed diffraction peaks confirm the coexistence of both $\mathrm{PbS}$ and $\mathrm{MoS}_{2}$ crystalline phases. However, the existence of multiple peaks suggests their polycrystalline nature. An intense peak at 14.1 degree for the (002) plane alongside four low intensity peaks at 33.1, 39.2, 57.5 and 61.2 degree is assigned to the (100), (103), (110) and (008) lattice planes of $\mathrm{MoS}_{2}$, respectively, indicating the formation of $2 \mathrm{H}$ semiconducting phase $\mathrm{MoS}_{2}$ (JCPDS file no. 89e2905) retaining crystalline quality and hexagonal symmetry, while diffraction peaks at 22.1, 26.2, 29.2, 44.3 and 50.5 degree representing the (110), (111), (200), (220) and (311) lattice planes of $\mathrm{PbS}$, respectively, agreed well with the cubic $\mathrm{PbS}$ structure (JCPDS file no. 20e0596). The intensity ratio of the (002) plane of $\mathrm{MoS}_{2}$ to that of the (200) plane of $\mathrm{PbS}$ in the $\mathrm{PbS} / \mathrm{MoS}_{2}$ hybrids reduces significantly with increasing $\mathrm{Pb}$ precursor concentration. The suppressed intensity is attributed to the increased size of PbS QDs, which wrap around the $\mathrm{MoS}_{2}$ surface, resulting in the attenuation of intensity. Room-temperature micro-Raman spectra of synthesized $\mathrm{PbS}$ decorated $\mathrm{MoS}_{2}$ hybrids for four different samples (S1-S4) are presented in Fig. 3(b) revealing the characteristic vibrational bands in the hybrids. The characteristic peaks of $\mathrm{MoS}_{2}$ at 379 and $404 \mathrm{~cm}^{-1}$ are ascribed to the inplane $\left(\mathrm{E}_{2 \mathrm{~g}}^{1}\right.$ band) and out-of-plane $\left(\mathrm{A}_{1 \mathrm{~g}}\right.$ band) vibrational modes, respectively. The spacing between two characteristic Raman shifts is about $\sim 25 \mathrm{~cm}^{-1}$, which is in agreement with the previously reported results corroborating the formation of few layer $\mathrm{MoS}_{2}$ nanosheets. ${ }^{30}$ Slightly upshifted and broadened Raman vibrational bands compared to the bulk $\mathrm{MoS}_{2}$ indicate the existence of substantial local defects and disorder in hybrids. The observation reveals that the local symmetry in the hybrid structure is different from that of the bulk $\mathrm{MoS}_{2}$, though the crystal structure remains unaltered. The other vibrational peaks are attributed to the stretching of diatomic molecules of $\mathrm{PbS}$ QDs. The weak and wide peak at $\sim 138 \mathrm{~cm}^{-1}$ corresponds to the combination of longitudinal and transverse acoustic modes [LA (A) + TA (L)], whereas, the strong one, near $\sim 280 \mathrm{~cm}^{-1}$ is attributed to the surface phonons (SP). A broad peak near $\sim 448 \mathrm{~cm}^{-1}$ is ascribed to the $2 \mathrm{LO}$ (longitudinal optical) phonon modes in $\mathrm{PbS}$ QDs, whereas the one, near $\sim 520 \mathrm{~cm}^{-1}$ is the contribution from the Si substrate. The increase in the vibrational peak intensity ratio of $\mathrm{PbS}$ to $\mathrm{MoS}_{2}$ as a function of $\mathrm{PbS}$ QDs is clear evidence of a larger fraction of $\mathrm{PbS}$ coverage on the $\mathrm{MoS}_{2}$ surface. All the vibrational modes of $\mathrm{PbS}$ are upshifted and broadened in hybrid nanostructures, as compared to that of 

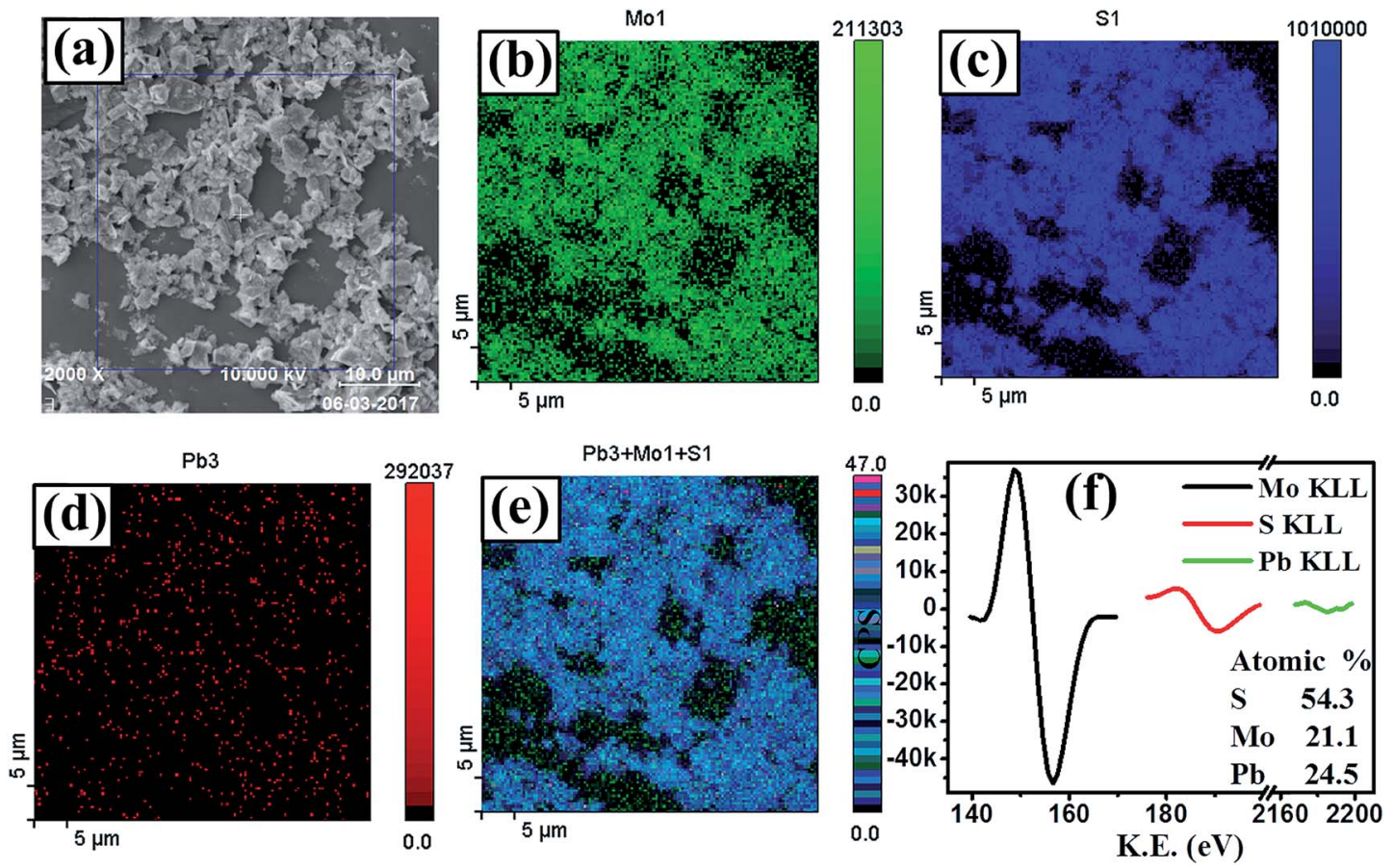

Fig. 2 (a) SEM micrograph of the as-synthesized PbS/MoS 2 hybrids (sample S1) used for Auger mapping. Auger electron spectroscopic images of the individual elementals showing the distribution of (b) Mo, (c) S and (d) Pb, along with (e) a combined elemental map. (f) Characteristic AES spectra of the elements present in sample-S1 to estimate their atomic percentage.

pristine PbS QDs reported in the literature. ${ }^{31,32}$ This is ascribed to the interaction between $\mathrm{PbS}$ and $\mathrm{MoS}_{2}$ via electron transfer and the associated strain due to structural defects and disorder.

Optical absorption spectra of as-prepared $\mathrm{PbS} / \mathrm{MoS}_{2}$ hybrid samples (S1-S4), recorded in the visible and IR range are shown in Fig. 4(a) and (b), respectively. The absorption spectra in the visible regime (Fig. 4(a)) exhibit two characteristic peaks at $606 \mathrm{~nm}(\sim 2.04 \mathrm{eV})$ and $665 \mathrm{~nm}(\sim 1.86 \mathrm{eV})$, which are attributed to the excitonic band edge transitions from spin-degenerate valence bands to the conduction band near the $K$-point in the Brillouin zone of $\mathrm{MoS}_{2}$. The appearance of an intense broader absorption peak around $\sim 400 \mathrm{~nm}(\sim 3.16 \mathrm{eV})$ is assigned to the band nesting transition arising in a localized region between $K$ and $\Gamma$-points in the band structure of $\mathrm{MoS}_{2}$ nanosheets. ${ }^{33}$ Notably, the optical absorption peak position in $\mathrm{MoS}_{2}$ nanosheets remains unaltered with the change of PbS QD size. The tunable absorption characteristics in the IR range from 1150 to $1350 \mathrm{~nm}$ with varying PbS QD size, shown in Fig. 4(b), are ascribed to the quantum confinement effect in PbS QDs. The near IR absorption peak, which is blue-shifted with decreasing PbS QD size, displays the size-tunable optical properties of PbS QDs. The results demonstrate the co-existence of optical signals from both $2 \mathrm{D}-\mathrm{MoS}_{2}$ and $0 \mathrm{D}-\mathrm{PbS}$ QDs, in the visible and IR region, respectively, making the $0 \mathrm{D} / 2 \mathrm{D}$ hybrids attractive for the fabrication of two color-band (visible broadband and tunable IR) photodetectors.

Photo-generated carrier lifetime in control $\mathrm{MoS}_{2}$ and $\mathrm{PbS} /$ $\mathrm{MoS}_{2}$ hybrid nanostructures was recorded at room temperature and the results are presented in Fig. 4(c). Experimental data for both the samples could be well fitted (solid lines) using the
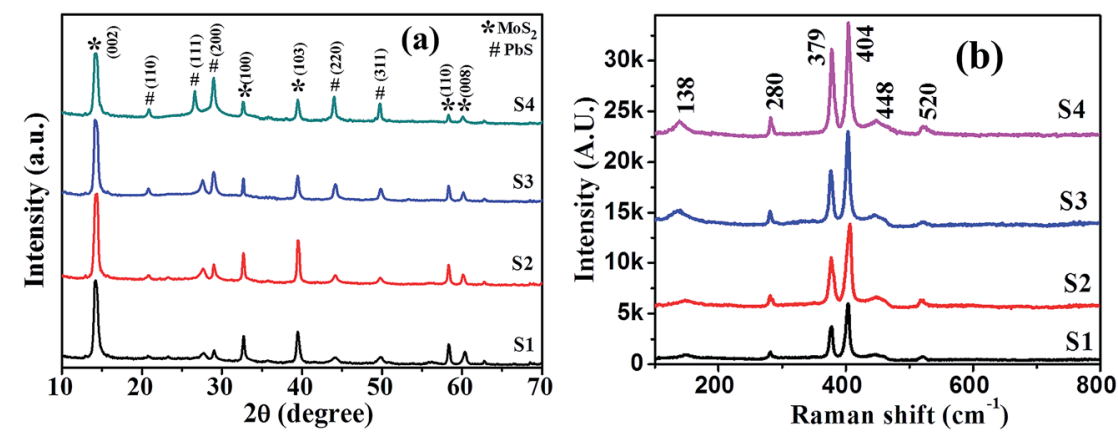

Fig. 3 (a) X-ray diffraction pattern and (b) Raman spectra of the samples revealing the simultaneous presence of 2D-MoS 2 and $0 D-P b S$ in hybrid nanostructures. 

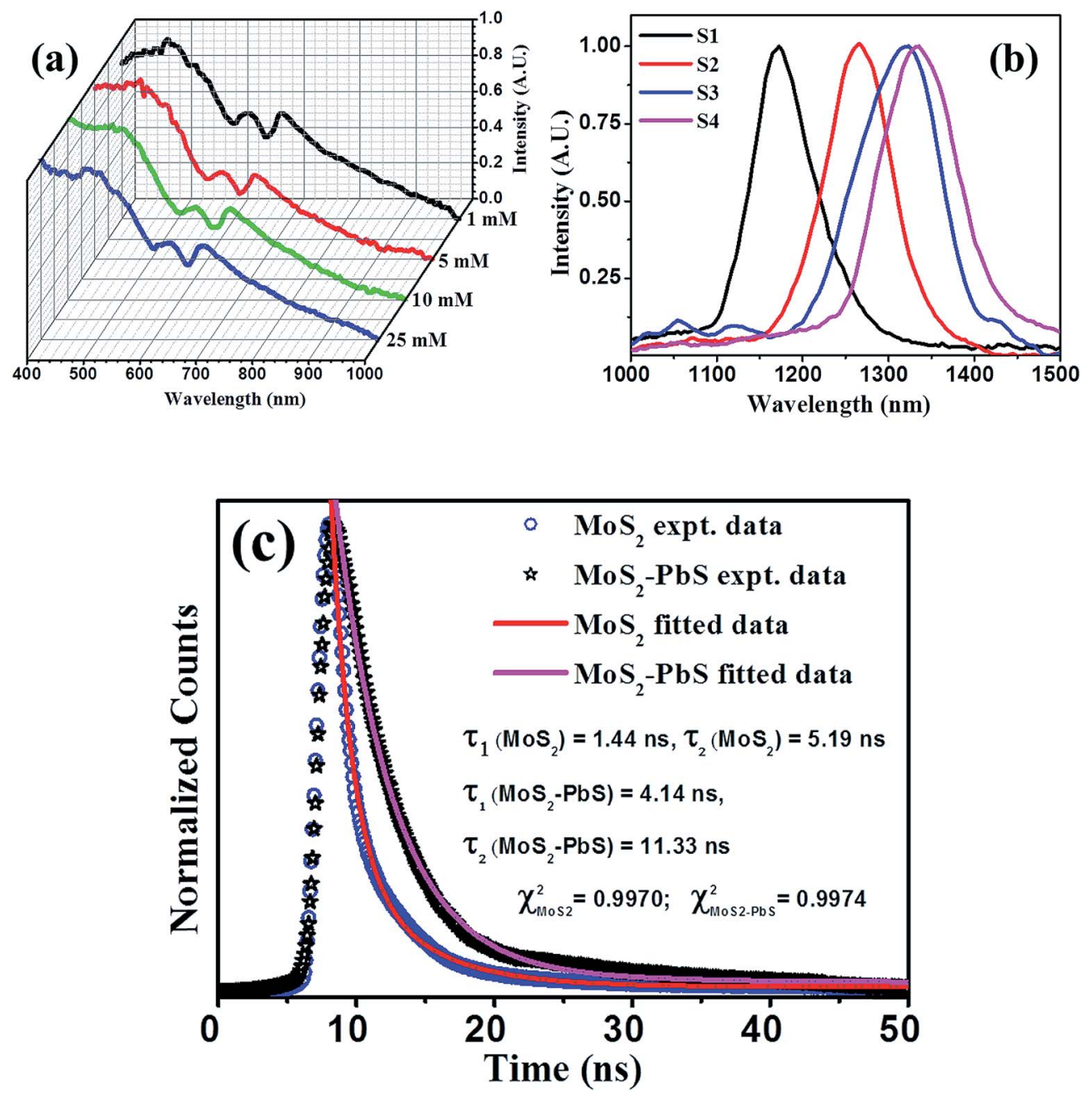

Fig. 4 Optical absorption spectra of hybrid samples for the (a) visible and (b) infrared color region, illustrating 2D-MoS ${ }_{2}$ excitonic absorption features with the signature of the direct band edge transition and size dependent absorption features for OD-PbS. (c) Time-resolved photoluminescence spectra of control $\mathrm{MoS}_{2}$ and $\mathrm{PbS} / \mathrm{MoS}_{2}$ hybrids with the latter showing a higher carrier lifetime. Solid lines are fit to the experimental data using eqn (1).

double exponent Marquardt-Levenberg algorithm, to compare and estimate the photogenerated carrier lifetime, given by, ${ }^{34}$

$$
I(t)=A_{1} \exp \left(-\frac{t}{\tau_{1}}\right)+A_{2} \exp \left(-\frac{t}{\tau_{2}}\right)
$$

where $I(t)$ is the luminescence intensity at time $t, A_{1}$ and $A_{2}$ are the decay coefficients, and $\tau_{1}$ and $\tau_{2}$ are the average lifetimes of bi-exponential decay. The estimated carrier life time is found to be $\tau_{1} \sim 1.44$ ns and $\tau_{2} \sim 5.19 \mathrm{~ns}$ for bare $\mathrm{MoS}_{2}$ nanosheets, while the same for the hybrid system is $\tau_{1} \sim 4.14 \mathrm{~ns}$ and $\tau_{2} \sim 11.33 \mathrm{~ns}$. The numerical fitting parameter, $\chi^{2}$, value is found to be $\sim 0.99$ for both the cases. These results are in qualitative agreement with the reported result for the PbS/RGO system..$^{35}$ The observed photo-carrier lifetimes are found to be much longer in the $\mathrm{PbS} /$ $\mathrm{MoS}_{2}$ hybrid nanostructures as compared to bare $\mathrm{MoS}_{2}$, which indicates that the electron-hole recombination process is delayed in the hybrid system. This is a signature of strong interaction between $\mathrm{PbS}$ QDs and the $\mathrm{MoS}_{2}$ layer with the transfer of photogenerated electrons from the conduction band of PbS QDs to that of $\mathrm{MoS}_{2}$, the details of which will be discussed later.

\subsection{Photodetection characteristics}

The device response of synthesized $\mathrm{PbS} / \mathrm{MoS}_{2}$ hybrid nanostructures has been studied by fabricating a flexible photodetector on a PET substrate. Digital photographs of the lithographically fabricated array of photodetectors are shown in Fig. 5(a). The details of substrate flexibility, transparency, device fabrication and indigenous testing set-up are presented in Fig. S4 (please see ESI $\dagger$ ). The room temperature current-voltage $(I-V)$ characteristics of the fabricated device for sample-S1 are illustrated in Fig. 5(b) under dark and illumination conditions. The symmetric $I-V$ nature establishes the formation of a backto-back junction between $\mathrm{Au}$ and $\mathrm{PbS} / \mathrm{MoS}_{2}$ hybrid nanostructures. A significant increase in the current level upon monochromatic illumination (@ $514 \mathrm{~nm}, 0.15 \mathrm{~mW} \mathrm{~cm}^{-2}$ ) is due to the generation of photo-carriers leading to a high photo-todark current ratio of the order of $\sim 10^{3}$ at a bias of $-2 \mathrm{~V}$. To 

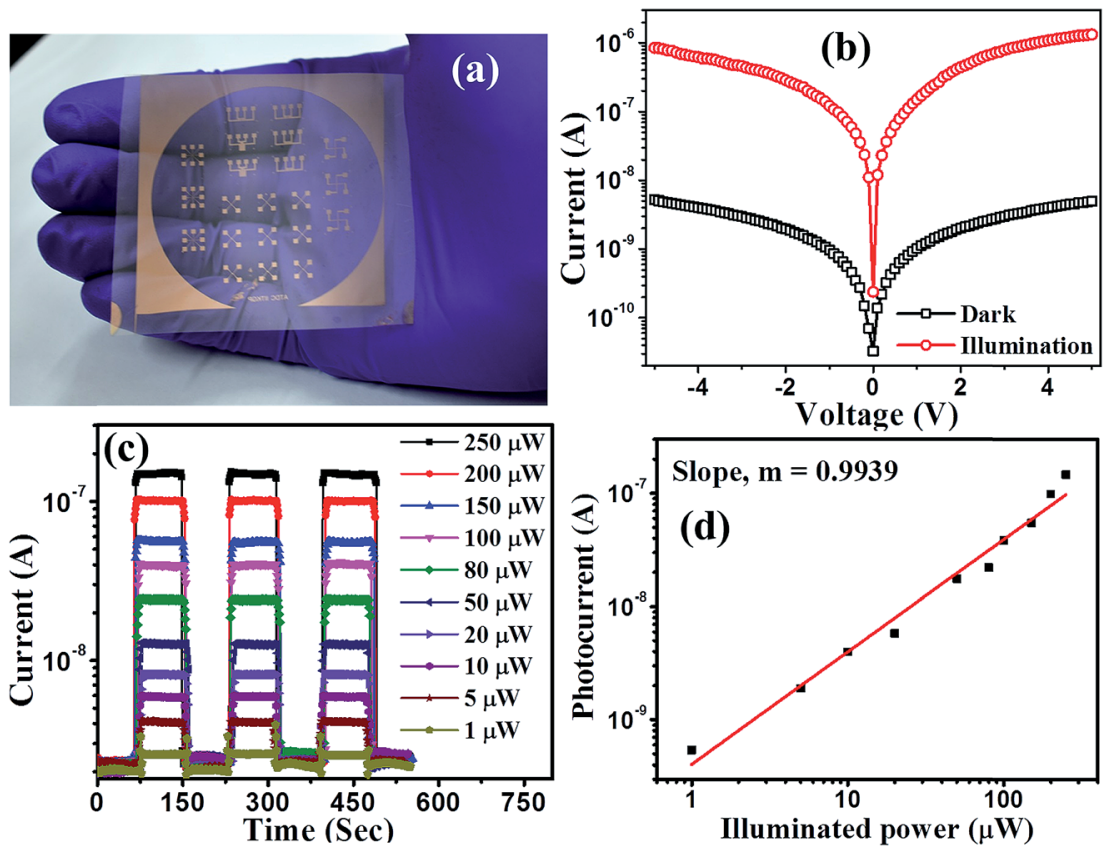

Fig. 5 (a) Digital photographs of metal (Au) electrodes patterned on transparent and flexible PET substrates fabricated using the photolithography technique. (b) Typical current-voltage characteristics of the as-fabricated device under dark and illumination conditions. (c) Temporal current response of the device for various illumination intensities. (d) Photocurrent as a function of illumination power showing a linear dependency.

study the photo-switching characteristics of the fabricated photodetector, the temporal photoresponse $(I-t)$ under a pulsed illumination (@ $\lambda=514 \mathrm{~nm}$ ) with various power densities at $2 \mathrm{~V}$ bias has been recorded and the results are presented in Fig. 5(c). The time-traces show a periodic and rapid alternate switching behavior of the current level upon pulsed illumination illustrating an excellent reproducibility, and a stable and reversible response of the photodetectors. An enhanced photo-current response has been observed with increasing the illumination intensity. The variation of photocurrent with incident optical power has been estimated and plotted in Fig. 5(d). The experimental data have been fitted using the power law $I_{\mathrm{Ph}} \propto P^{z}$, where the exponent " $z$ " represents the rate of generation and recombination of electron-hole pairs leading to resultant photocurrent. ${ }^{36}$ The photocurrent $\left(I_{\mathrm{Ph}}\right)$ increases monotonically with incident optical power $(P)$ suggesting that the generation rate of carriers is proportional to the absorbed photon flux. Therefore, the responsivity is constant throughout the measured power density regime, making the device attractive for practical applications.

The estimated spectral responsivity of the hybrid photodetector in the wavelength range from 400 to $1600 \mathrm{~nm}$ is presented in Fig. 6(a), for four different PbS QD sizes (S1-S4). The typical spectrum exhibits a broadband spectral response with the detection range extending to the IR region. The estimated peak response for the device with sample-S1 is found to be $\sim 398 \mathrm{~mA} \mathrm{~W}^{-1}$ for $480 \mathrm{~nm}$ and $\sim 543 \mathrm{~mA} \mathrm{~W}^{-1}$ for $1200 \mathrm{~nm}$ at $2 \mathrm{~V}$. The responsivity value is almost constant in the visible wavelength range of 480 to $660 \mathrm{~nm}$. On the other hand, the responsivity peak in the IR regime is ascribed to the optical transitions in PbS QDs under illumination. The photoresponse peak in the IR wavelength range exhibits a red shift with increasing PbS QD size, which could be useful to fabricate a wavelength selective IR detector. The responsivity spectra of fabricated detectors closely resemble the absorption characteristics of hybrid materials, indicating that the spectral selectivity is determined by the size induced bandgap of PbS QDs.

It is interesting to note that the hybrid device fabricated with the lowest QDs size (sample-S1) shows the highest responsivity, as shown in Fig. 6(a). The responsivity in both visible and IR regions decreases with increasing size of PbS QDs. This can be explained by the reduced carrier recombination lifetime in larger sized PbS QDs. The specific detectivity $\left(D^{*}\right)$ of a detector, the ability to differentiate the minimum optical signal power from the background noise, has been estimated as a function of wavelength over the $400-1600 \mathrm{~nm}$ range and is plotted in Fig. 6(b). The spectra show a peak detectivity of $\sim 2 \times 10^{12}$ Jones around $500 \mathrm{~nm}$ and $\sim 2.68 \times 10^{12}$ Jones around $1200 \mathrm{~nm}$ for $2 \mathrm{~V}$ bias, much higher than those of the standard Si or Ge based detectors. ${ }^{36,37}$ A comparison of the responsivity, detectivity and operation wavelength region of representative photodetectors fabricated using a similar type of colloidal QDs is provided in Table 1. It may be noted that our results are comparable or superior in terms of responsivity, detectivity and spectral wavelength coverage when compared with the all-solution processed colloidal QD based system as shown in Table 1.

A combination of higher responsivity, lower dark current and lower noise levels leads to superior detectivity in $0 \mathrm{D}-2 \mathrm{D}$ hybrids. The detectivity is found to be significantly enhanced and the peak is blue shifted with the reduction of PbS QD size, similar to 

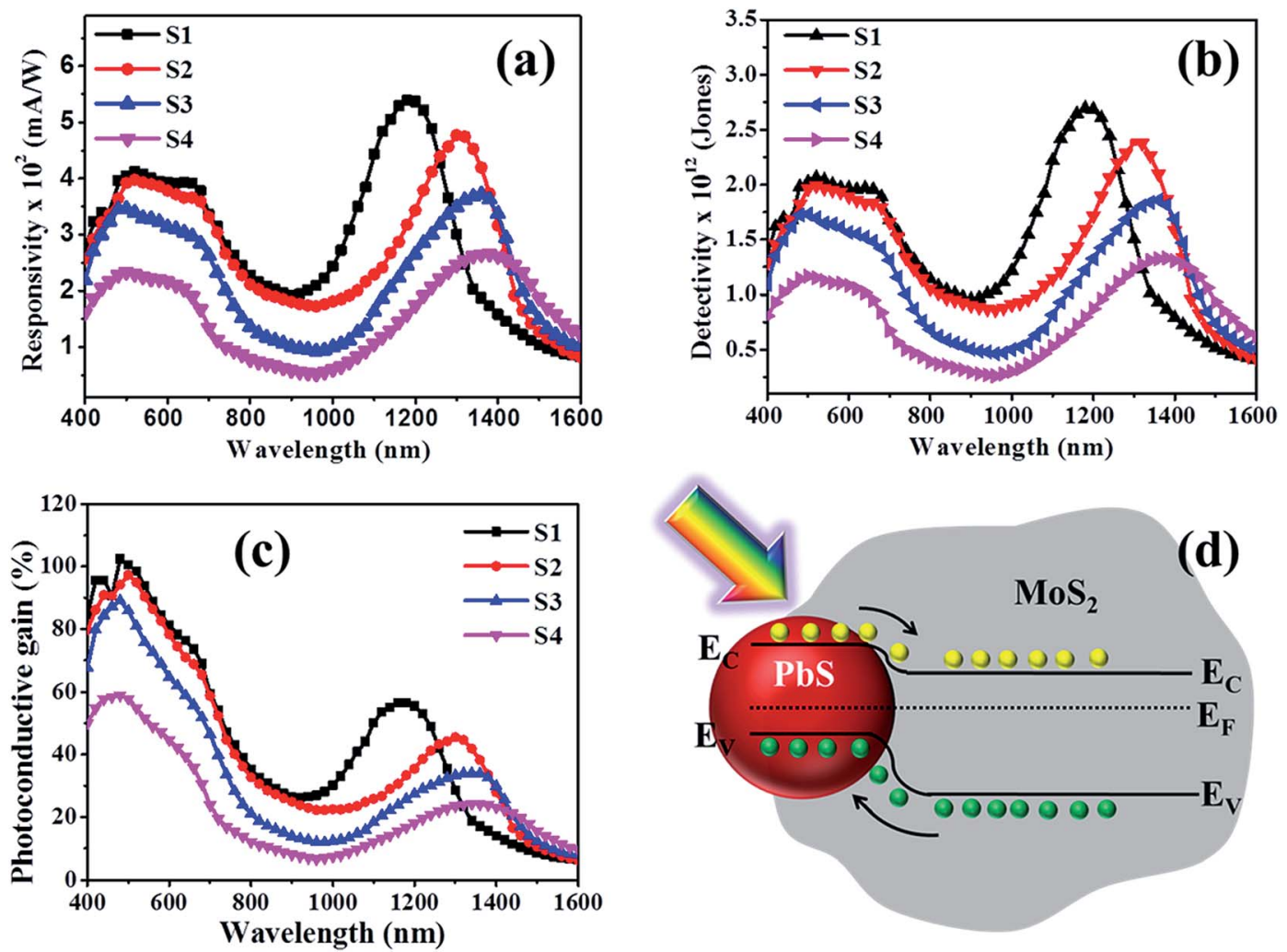

Fig. 6 (a) Spectral responsivity, (b) detectivity and (c) photoconductive gain of the fabricated heterostructure for different samples, recorded at an applied bias of $2 \mathrm{~V}$ with $100 \mu \mathrm{W}$ optical power. (d) Estimated band edge alignment of $\mathrm{n}$-type $\mathrm{MoS}_{2}$ and $\mathrm{p}$-type PbS showing the formation of a type-II heterojunction under equilibrium conditions.

the responsivity behaviour. The photoconductive gain, defined as the ratio of collected carriers per incident photon per unit time, is related to the electronic transport and the carrier collection efficiency of a detector has been studied. The wavelength dependent photoconductive gain $\left(G_{\mathrm{Ph}}\right)$ is shown in Fig. 6(c). The figure illustrates that the smallest size PbS QD based device (S1) displays the highest gain, which is nearly $100 \%$ ( 1 electron collected per incident photon) at $\sim 500 \mathrm{~nm}$. The relatively high photoconductive gain in the device originates from the synergistic effect of $\mathrm{PbS} / \mathrm{MoS}_{2}$, where the $\mathrm{MoS}_{2}$ channel with high carrier mobility provides faster transfer of photogenerated electrons. Moreover, the longer carrier recombination lifetime in the $2 \mathrm{D}-0 \mathrm{D}$ hybrids is also beneficial for achieving a higher gain. At a higher bias, the photogenerated carriers are collected more efficiently, yielding a higher gain. However, for the highest PbS QD size (sample-S4), the photoresponse is found to be degraded. A combined effect of the attenuated illumination for higher PbS coverage, increased surface roughness scattering of carriers and reduced surface area contributes to the suppressed gain value. The improved photoresponse of the fabricated devices originates from the synergistic interaction of carriers between PbS QDs and $\mathrm{MoS}_{2}$ nanosheets. When p-type PbS QDs are in contact with n-type $\mathrm{MoS}_{2}$, surface electrons of $\mathrm{MoS}_{2}$ diffuse towards the PbS QDs until a built-in potential is formed at the interface and Fermi levels are in equilibrium. Consequently, the electron transfer

Table 1 Comparison of the device performances based on solution-processed colloidal QDs

\begin{tabular}{|c|c|c|c|c|}
\hline Sample description & Responsivity $\left(\mathrm{A} \mathrm{W}^{-1}\right)$ & Detectivity (Jones) & $\begin{array}{l}\text { Operational } \\
\text { spectral range }\end{array}$ & Ref. \\
\hline $\mathrm{PbS}_{0.4} \mathrm{Se}_{0.6}$ colloidal quantum dot film & 0.05 & $4.11 \times 10^{9}$ & $980 \mathrm{~nm}$ & 38 \\
\hline \multirow{2}{*}{ PbS QDs/ZnO heterostructures } & 2.73 & $2.39 \times 10^{12}$ & $532 \mathrm{~nm}$ & 39 \\
\hline & 0.42 & $3.65 \times 10^{11}$ & $808 \mathrm{~nm}$ & \\
\hline $\mathrm{MoS}_{2}$ nanocrystal film & 0.13 & - & $400-700 \mathrm{~nm}$ & 4 \\
\hline HgTe QD capped with DDT ligands & 0.01 & - & $1620 \mathrm{~nm}$ & 40 \\
\hline PbS colloidal QDs & 0.2 & $10^{12}$ & $1550 \mathrm{~nm}$ & 41 \\
\hline HgSe colloidal QD film & 0.8 & $10^{8}$ & $3000-8000 \mathrm{~nm}$ & 42 \\
\hline PbS quantum dots with oleic acid capped & 0.2 & - & $1300-1500 \mathrm{~nm}$ & 43 \\
\hline PbS QDs/MoS ${ }_{2}$ hybrid heterostructure & 0.6 & $10^{12}$ & $400-1600 \mathrm{~nm}$ & This work \\
\hline
\end{tabular}



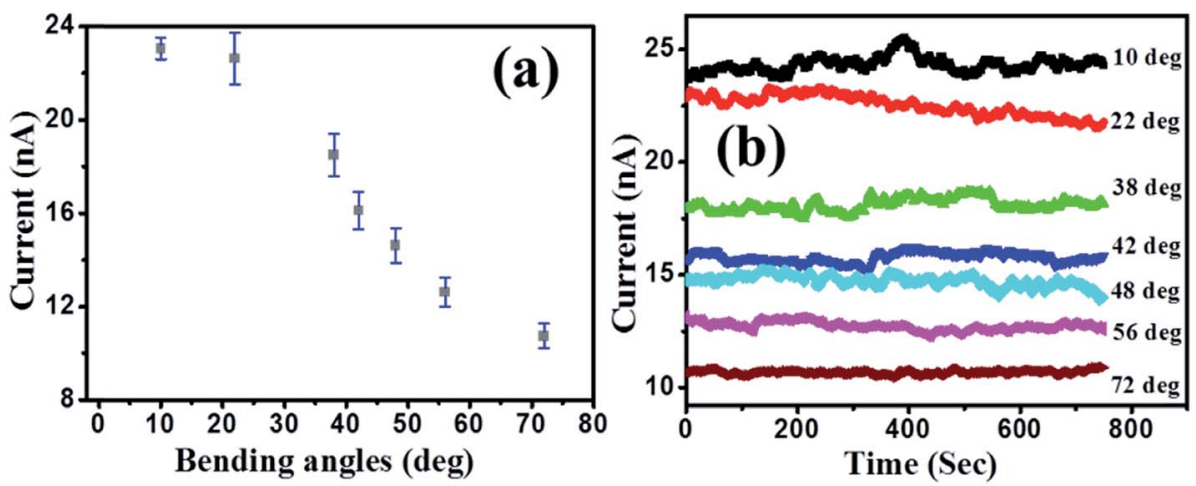

Fig. 7 Dependence of the output dark current level on the (a) bending angle and (b) time for a fixed bending angle.

reduces the energy level of the conduction band producing a type-II band alignment between $\mathrm{n}-\mathrm{MoS}_{2}$ and $\mathrm{p}-\mathrm{PbS}$ QDs. The resultant band diagram, under equilibrium conditions, is shown in Fig. 6(d). When the PbS/MoS 2 hybrids are illuminated, the photogenerated electrons in PbS are easily transferred to the conduction band of $\mathrm{MoS}_{2}$, while holes move in the opposite direction. The enhanced photoresponse of the detector with applied bias is corroborated by the efficient collection of photocarriers at the electrodes in the presence of an electric field, resulting in a higher photocurrent (please see Fig. S5 in the ESI $\dagger$ ). The most striking feature of the device is the combination of a high efficiency with a broadband spectral response in the visible region and tunable detection in the IR region.

The solution-based fabrication of hybrids is compatible with flexible platforms making it attractive for various applications. In order to study the effect of mechanical stress (bending) on the output characteristics, $\mathrm{PbS} / \mathrm{MoS}_{2}$ hybrid devices have been fabricated on PET substrates. The dependence of the output current on the bending angle over various bending cycles has been studied and the results are presented in Fig. 7(a) and (b). The output current does not degrade with increasing bending angles up to 20 degree, as shown in Fig. 7(a). On the other hand, Fig. 7(b) displays an excellent stability of the current level over a fixed bending condition, which establishes the efficacy of the hybrid device for use as a flexible photodetector. This observation confirms the reproducibility and the repeatability of the device performance after recovering from a stressed condition. To the best of our knowledge, this is the first report on solution processed, IR absorbing QD decorated $\mathrm{MoS}_{2}$ devices, which can respond to two different colour bands ranging from 400$1600 \mathrm{~nm}$ and is compatible with flexible platforms.

\section{Conclusions}

We have demonstrated a novel and catalyst/stabilizer free, onepot synthesis process of $\mathrm{PbS} / \mathrm{MoS}_{2}$ hybrid nanostructures using a low temperature solvothermal method, which is promising for the fabrication of cost-effective, large area, two-color and infrared tunable wide-band photodetectors. The integration of 0D-PbS with 2D-MoS ${ }_{2}$ provides two significant advantages: (1) $\mathrm{MoS}_{2}$ acts as a substrate for nucleation and growth of PbS QDs and at the same time hinders their agglomeration. (2) The photo-excited electron-hole pairs in the hybrids could be efficiently separated in type-II heterostructures resulting in a higher photocurrent and gain. The device fabricated using the $\mathrm{PbS}$ QDs/ $\mathrm{MoS}_{2}$ hybrid heterostructure demonstrates two colour detection with a broad spectral responsivity in the visible region and a tunable response in the IR region. The performance of the fabricated devices in the infrared region is significantly superior to that of their analogues with hybrid structures and even better than the performance of commercial Si-, Ge- and InGaAs-based devices. The solution processability of colloidal synthesis offered monolithic integration of devices on flexible substrates. The experimental findings suggest that the synergic $0 \mathrm{D} / 2 \mathrm{D}$ hybrid heterostructure devices exhibit superior responsivity compared to solution processable devices achieved individually using PbS QDs or $\mathrm{MoS}_{2}$. This result could pave the way for the successful integration of 0D QDs in 2D materials toward novel and high performance next-generation photonic devices, compatible with standard CMOS technology as well as flexible platforms.

\section{Conflicts of interest}

The authors declare no conflict of interest.

\section{Acknowledgements}

The authors would like to acknowledge the partial financial support of the "NNETRA-SWI" project (Grant No. DST/NM/ NNETRA/2018(G)) sponsored by Meity and DST, Govt. of India and the DST-CERI supported "ICF" Project at IIT Kharagpur (Grant No. DST/TM/CERI/C74(G)).

\section{References}

1 K. F. Mak, C. Lee, J. Hone, J. Shan and T. F. Heinz, Phys. Rev. Lett., 2010, 105, 136805.

2 H. S. Lee, S. S. Baik, K. Lee, S. W. Min, P. J. Jeon, J. S. Kim, K. Choi, H. J. Choi, J. H. Kim and S. Im, ACS Nano, 2015, 9, 8312-8320.

3 A. Splendiani, L. Sun, Y. Zhang, T. Li, J. Kim, C. Y. Chim, G. Galli and F. Wang, Nano Lett., 2010, 10, 1271-1275. 
4 S. Mukherjee, R. Maiti, A. Midya, S. Das and S. K. Ray, ACS Photonics, 2015, 2, 760-768.

5 A. Ebnonnasir, B. Narayanan, S. Kodambaka and C. V. Ciobanu, Appl. Phys. Lett., 2014, 105, 031603.

6 K. Koåmider and J. Fernández-Rossier, Phys. Rev. B: Condens. Matter Mater. Phys., 2013, 87, 075451.

7 D. Jariwala, T. J. Marks and M. C. Hersam, Nat. Mater., 2016, 16, 170.

8 Y. Gong, J. Lin, X. Wang, G. Shi, S. Lei, Z. Lin, X. Zou, G. Ye, R. Vajtai, B. I. Yakobson, H. Terrones, M. Terrones, B. K. Tay, J. Lou, S. T. Pantelides, Z. Liu, W. Zhou and P. M. Ajayan, Nat. Mater., 2014, 13, 1135.

9 C. Li, X. Yan, X. Song, W. Bao, S. Ding, D. W. Zhang and P. Zhou, Nanotechnology, 2017, 28, 415201.

10 Y. Chen, X. Wang, G. Wu, Z. Wang, H. Fang, T. Lin, S. Sun, H. Shen, W. Hu, J. Wang, J. Sun, X. Meng and J. Chu, Small, 2018, 14, 1703293.

11 A. Midya, A. Ghorai, S. Mukherjee, R. Maiti and S. K. Ray, J. Mater. Chem. A, 2016, 4, 4534-4543.

12 V. Dhyani and S. Das, Sci. Rep., 2017, 7, 44243.

13 S. Mukherjee, S. Biswas, S. Das and S. K. Ray, Nanotechnology, 2017, 28, 135203.

14 R. Zhuo, Y. Wang, D. Wu, Z. Lou, Z. Shi, T. Xu, J. Xu, Y. Tiana and X. Li, J. Mater. Chem. C, 2018, 6, 299-303.

15 A. Tayyebi, M. M. Tavakoli, M. Outokesh, A. Shafiekhani and A. A. Simchi, Ind. Eng. Chem. Res., 2015, 54, 7382-7392.

16 X. Song, Y. Zhang, H. Zhang, Y. Yu, M. Cao, Y. Che, H. Dai, J. Yang, X. Ding and J. Yao, Nanotechnology, 2017, 28, 145201.

17 W. Feng, C. Qin, Y. Shen, Y. Li, W. Luo, H. An and Y. Feng, Sci. Rep., 2014, 4, 3777.

18 V. R. Raja, D. R. Rosaline, A. Suganthi and M. Rajarajan, Solid State Sci., 2017, 67, 99-108.

19 S. Cho, Y. Jo, H. Woo, J. Kim, J. Kwak, H. Kim and H. Im, Applied Science and Convergence Technology, 2017, 26, 47-49.

20 D. Kufer, I. Nikitskiy, T. Lasanta, G. Navickaite, F. H. L. Koppens and G. Konstantatos, Adv. Mater., 2015, 27, 176-180.

21 H. Zhao, D. Wang, M. Chaker and D. Ma, J. Phys. Chem. C, 2011, 115, 1620-1626.

22 M. Peng, X. Xie, H. Zheng, Y. Wang, Q. Zhuo, G. Yuan, W. Ma, M. Shao, Z. Wen and X. Sun, ACS Appl. Mater. Interfaces, 2018, 10, 43887-43895.

23 G. Flora, D. Gupta and A. Tiwari, Interdiscip. Toxicol., 2012, 5, 47-58.

24 R. Saran and R. J. Curry, Nat. Photonics, 2016, $10,81$.

25 S. Mukherjee, S. Biswas, A. Ghorai, A. Midya, S. Das and S. K. Ray, J. Phys. Chem. C, 2018, 122, 12502-12511.
26 S. Acharya, D. D. Sarma, Y. Golan, S. Sengupta and K. Ariga, J. Am. Chem. Soc., 2009, 131, 11282-11283.

27 N. M. D. Brown, N. Cui and A. Mckinley, Appl. Surf. Sci., 1998, 134, 11-21.

28 N. P. Kondekar, M. G. Boebinger, E. V. Woods and M. T. McDowell, ACS Appl. Mater. Interfaces, 2017, 9, 32394-32404.

29 J. D. Patel, F. Mighri, A. Ajji and S. Elkoun, Mater. Sci. Appl., 2012, 3, 125-130.

30 C. Lee, H. Yan, L. E. Brus, T. F. Heinz, J. Hone and S. Ryu, ACS Nano, 2010, 4, 2695-2700.

31 A. Milekhin, L. Sveshnikova, T. Duda, N. Surovtsev, S. Adichtchev, L. Ding and D. R. T. Zahn, J. Vac. Sci. Technol., B: Microelectron. Nanometer Struct.-Process., Meas., Phenom., 2010, 28, C5E22-C5E24.

32 A. V. Baranov, K. V. Bogdanov, E. V. Ushakova, S. A. Cherevkov, A. V. Fedorov and S. Tscharntke, Opt. Spectrosc., 2010, 109, 268-271.

33 S. H. Aleithan, M. Y. Livshits, S. Khadka, J. J. Rack, M. E. Kordesch and E. Stinaff, Phys. Rev. B, 2016, 94, 035445 .

34 S. Mukherjee, R. Maiti, A. K. Katiyar, S. Das and S. K. Ray, Sci. Rep., 2016, 6, 29016.

35 B. Martín-García, A. Polovitsyn, M. Prato and I. Moreels, J. Mater. Chem. C, 2015, 3, 7088-7095.

36 S. Mukherjee, K. Das, S. Das and S. K. Ray, ACS Photonics, 2018, 5, 4170-4178.

37 K. Das, S. Mukherjee, S. Manna, S. K. Ray and A. K. Raychaudhuri, Nanoscale, 2014, 6, 11232-11239.

38 S. Muhammad, S. Yang, T. Song, H. Wang, Y. Wang, B. He, M. Dong, Y. Tang, Y. Song and B. Zou, RSC Adv., 2016, 6, 87730-87737.

39 M. Peng, Y. Wang, Q. Shen, X. Xie, H. Zheng, W. Ma, Z. Wen and X. Sun, Sci. China Mater., 2019, 62, 225-235.

40 Z. Bingqing, M. Chen, S. V. Kershaw, A. L. Rogach, N. Zhao and H. K. Tsang, Conference on Lasers and Electro-Optics Pacific Rim (CLEO-PR), IEEE, 2017, pp. 1-3.

41 C. P. Jason, G. Konstantatos, K. W. Johnston, S. Hoogland, L. Levina and E. H. Sargent, Nat. Nanotechnol., 2009, 4, 40.

42 L. Emmanuel, M. Scarafagio, P. Hease, B. Nadal, H. Aubin, X. Z. Xu, N. Lequeux, G. Patriarche, S. Ithurria and B. Dubertret, Nano Lett., 2016, 16, 1282-1286.

43 M. Pawel, E. Georgitzikis, J. Maes, I. Vamvaka, F. Frazzica, J. V. Olmen, P. D. Moor, P. Heremans, Z. Hens and D. Cheyns, Sensors, 2017, 17, 2867. 